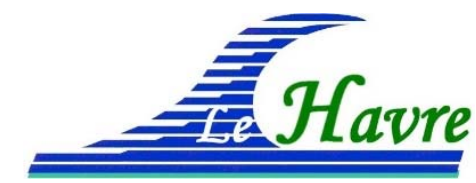

XVI èmes Journées Nationales Génie Côtier - Génie Civil

Le Havre, 2020

DOI:10.5150/jngcgc.2020.043 C Editions Paralia CFL

disponible en ligne - http://www.paralia.fr - available online

\title{
SEABIM - outil de numérisation du contrôle de digue en blocs préfabriqués
}

\author{
Steven LE BARS ${ }^{1}$, Vincent MACAIGNE ${ }^{1}$, Timothée LAUNAY ${ }^{1}$
}

1. ID OCEAN, Hangar D20, Village des Mascareignes 2, 97420 Le Port, Réunion, France.

contact@idocean.re

\section{Résumé :}

Afin de réaliser le contrôle et le suivi de la partie immergée de la digue en éléments préfabriqués de type ACCROPODETM II de la Nouvelle Route du Littoral à La Réunion, l'outil informatique SEABIM a été développé. Il permet de reconstruire un modèle 3D contenant l'ensemble des éléments préfabriqués d'une digue à partir d'un nuage de points (bathymétrie multifaisceaux pour la partie immergée et photogrammétrie drone en aérien), afin de créer un jumeau numérique de l'ouvrage. La précision du recalage (écarts entre les points et le modèle) est équivalente à la précision relative du système d'acquisition, de l'ordre de $2 \mathrm{~cm}$ dans notre cas avec un sondeur multifaisceau haute résolution, ce qui permet d'appliquer des contrôles automatisés de qualité de pose et d'identifier les anomalies. De plus, la superposition de modèles réalisés à des instants différents permet d'analyser l'évolution de l'ouvrage et de planifier des inspections complémentaires localisées ou des interventions de réparation. Aucun modèle initial, issu par exemple de données de pose, n'est nécessaire au recalage des éléments. Ainsi le jumeau numérique d'une digue existante peut être créé grâce à une simple acquisition de nuage de points haute densité afin d'en réaliser l'état zéro ou antérieur.

L'outil développé est utilisé par les ingénieurs contrôle interne du groupement en charge de la réalisation de la digue de la NRL.

\section{Mots-clés :}

Digue, Nouvelle Route du Littoral, Instrumentation, Bathymétrie, Inspection, Travaux maritimes.

\section{Introduction}

Le contrôle de digue monocouche en blocs béton préfabriqués peut s'avérer difficile pour les ingénieurs tant à la pose qu'en suivi temporel, d'autant plus sur la partie sous-marine des ouvrages. En effet, ce type de construction côtière requière la pose d'environ six mille unités par kilomètre d'ouvrage. Il est donc nécessaire de disposer d'outils efficaces et précis de contrôle de ces ouvrages afin d'en assurer la pérennité à long terme face aux forçages hydrauliques. Par conséquent, la solution de contrôle numérique de digue SEABIM a été mise en place dans le cadre du chantier de la Nouvelle Route du Littoral à La Réunion (NRL) afin d'assurer le suivi de la digue après sa construction. La NRL est 


\section{Thème 3 - Instrumentation, mesures, imagerie et télédétection}

construite sur cinq digues pour une longueur totale de $6,7 \mathrm{~km}$ par le groupement GTOI (Bouygues), SBTPC et VCT (Vinci). Ces digues sont couplées à un viaduc en mer de 5,4 kms afin de relier la capitale Saint-Denis et la ville de La Possession.
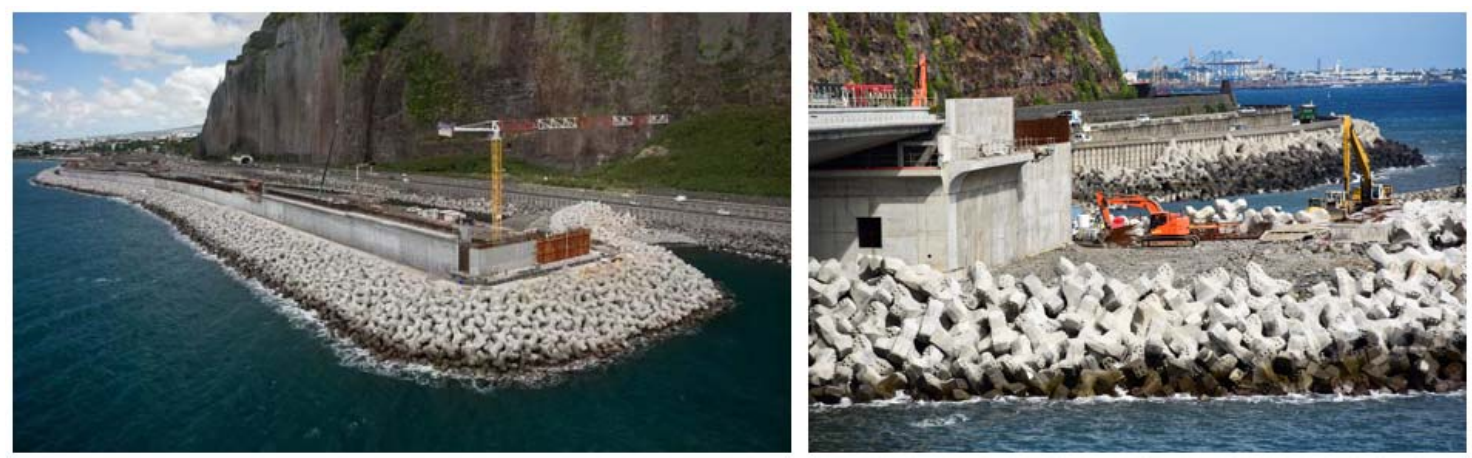

Figure 1. Digue D1 de la NRL.

Ces digues sont protégées par une monocouche d'éléments préfabriqués de type ACCROPODE ${ }^{\mathrm{TM}}$ II qui sont posés sur la sous-couche selon des règles de pose définies par le concepteur afin d'assurer la résistance structurelle de l'ouvrage face aux sollicitations de houle qui heurtent le littoral de l'île classée en zone cyclonique.
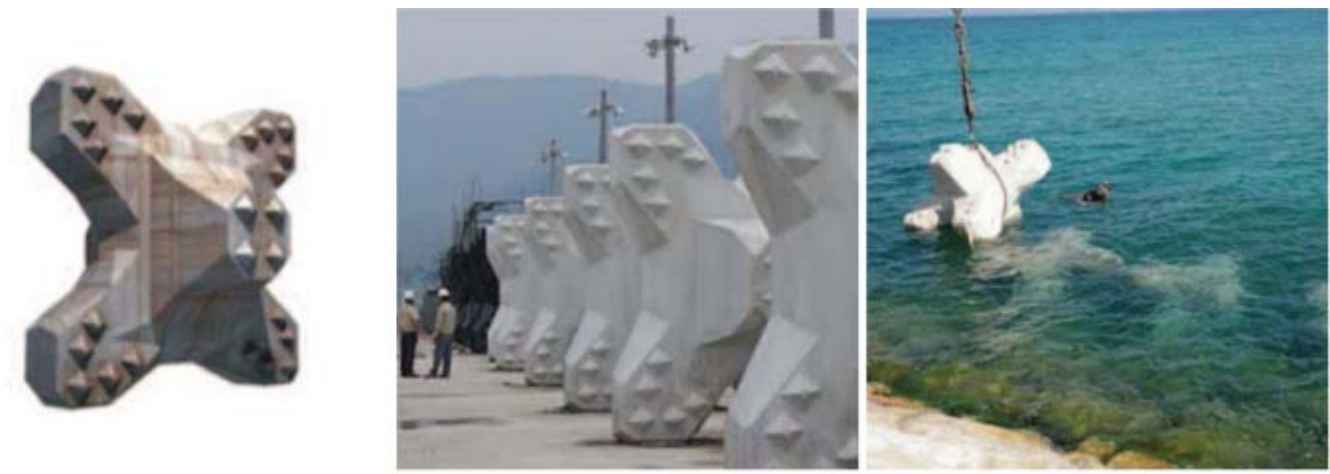

Figure 2. ACCROPODE ${ }^{\mathrm{TM}}$ II utilisé sur la NRL.

Généralement le contrôle de ces ouvrages est réalisé par bathymétrie multifaisceaux pour la partie sous-marine et par l'obtention d'un nuage de points par photogrammétrie ou par lidar en aérien. Des contrôles vidéo sous-marins de tout l'ouvrage sont ensuite réalisés par des plongeurs.

L'interprétation de ces données requière un investissement en temps conséquent pour les ingénieurs en charge du contrôle des ouvrages et rend difficile l'analyse objective de certaines situations critiques. Afin de palier à cette difficulté, un algorithme de détection automatique de blocs dans l'enveloppe du nuage de points a été développé afin de créer un jumeau numérique 3D tel que construit de la digue. Celui-ci permet de fournir une vue d'ensemble de l'ouvrage sur un logiciel spécialisé et facilement lisible par tous les acteurs 


\section{XVIèmes Journées Nationales Génie Côtier - Génie Civil \\ Le Havre 2020}

du projet. On peut ainsi identifier les anomalies structurelles et planifier des interventions complémentaires de contrôle sous-marin localisées sur les zones critiques dans une approche BIM (Building Information Modeling) dérivée du secteur BTP.
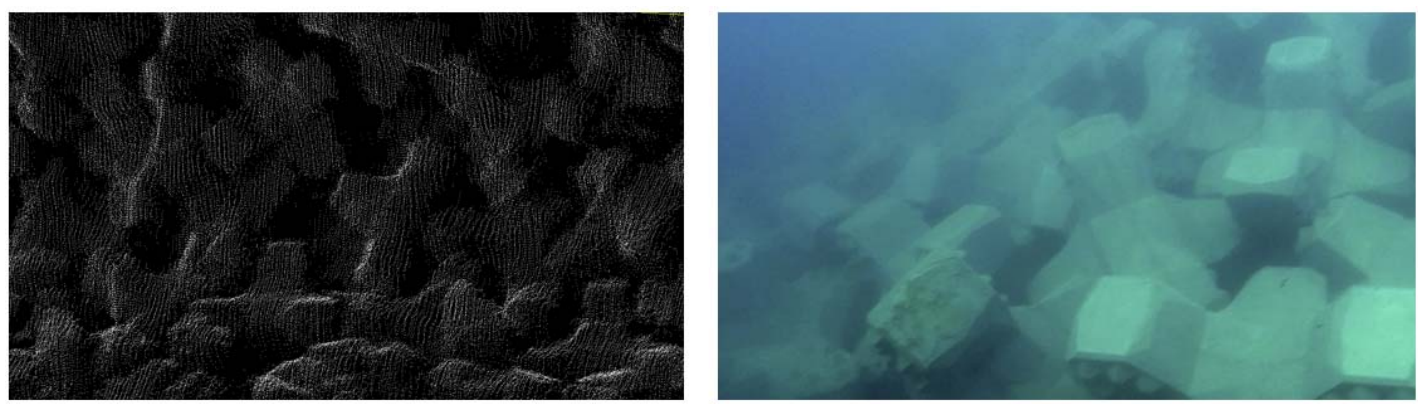

Figure 3. Rendu bathymétrique multifaisceaux à gauche, inspection plongeur à droite.

\section{L'outil SEABIM}

\subsection{Les règles de pose}

Selon le concepteur des digues en ACCROPODES ${ }^{\mathrm{TM}}$ II, les règles de pose suivantes doivent être respectées pour assurer la réception de l'ensemble de la carapace (CLI, 2018) :

- Densité de pose entre 95 et $105 \%$;

- Blocs posés en une couche sans hors profil (1/3 du bloc en dehors de la carapace);

- Blocs imbriqués sans liberté de mouvement ;

- Maillage losange des blocs globalement présent;

- Attitude variée des blocs ;

- Impossibilité pour un bloc de sous-couche de sortie par une aération.

Etant difficile pour un non expert d'analyser de manière objective ces données par une inspection sous-marine par plongeur ou via un nuage de points, l'objectif du projet de $\mathrm{R} \& \mathrm{D}$ est de créer un modèle numérique $3 \mathrm{D}$ de la digue de manière automatisée et d'appliquer des filtres de contrôle qui permettent de valider ces critères de pose.

\subsection{Création d'un jumeau numérique de digue}

Les données d'entrée utilisées pour l'algorithme de recalage de blocs dans le nuage de points sont de type données bathymétriques multifaisceaux haute résolution pour la partie sous-marine et photogrammétrie aérienne par drone RTK ou lidar depuis un navire pour la partie terrestre. La zone de ligne d'eau contient souvent des données de mauvaise qualité car les sondeurs bathymétriques couvrent mal cette zone (formation de bulles d'air liées aux vagues déferlantes, limite d'ouverture des sondeurs) surtout s'ils ne sont pas montés avec une orientation mécanique orientée vers la digue lors de l'acquisition. Cette zone peut être complétée en jouant sur le marnage à différentes heures d'acquisition pour 


\section{Thème 3 - Instrumentation, mesures, imagerie et télédétection}

les zones à forte marée afin de faire l'acquisition des lignes concernées hors d'eau par photogrammétrie drone. Pour les zones à marnage faible comme c'est le cas à La Réunion ( $0.5 \mathrm{~m}$ environ), le nuage de points au niveau de la ligne d'eau est acquis par une méthodologie mise en place pour le projet de reconstitution photogrammétrique par des photos prises depuis une caméra montée sur perche immergée sous la ligne d'eau et couplée à un GPS RTK.

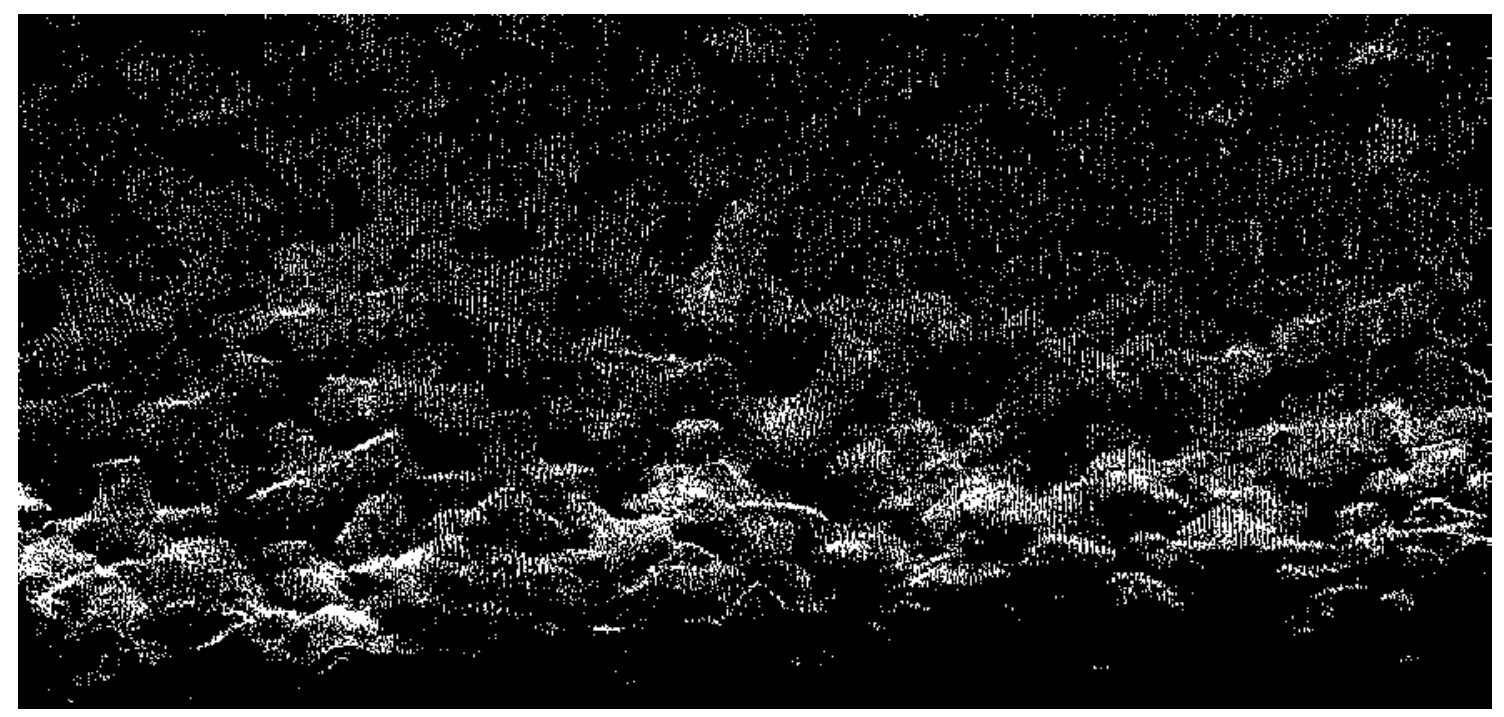

Figure 1. Etape 1 - Nuage de points d'entrée de l'algorithme.

La précision d'acquisition des données bathymétriques dépend du matériel utilisé, notamment de la qualité de la centrale d'altitude du système multifaisceaux et de son intégration ainsi que de la précision GPS. Dans le cadre du projet NRL, les données sousmarines sont acquises à l'aide d'un système haute résolution dont la précision absolue observée du nuage de points obtenue est inférieure à $5 \mathrm{~cm}$ en 3D. Pour les données aériennes, des levés 3D par photogrammétrie drone sont utilisés dont la précision absolue dépend de la qualité du référencement du modèle par point de contrôle au sol au GPS RTK. La précision absolue observée du modèle aérien généré est de $5 \mathrm{~cm}$. Les données aériennes issues du Lidar embarqué sur le navire ont posé plus de difficultés car celles-ci présentent un effet de double peau sur le nuage de points généré dû à un matériel utilisé de qualité insuffisante pour un levé précis, ces données n'ont donc pas été utilisées après analyse. A partir des données acquises sur le terrain qui nous ont été directement fournies par le groupement Digues NRL qui est en charge de ses propres acquisitions, un procédé algorithmique de reconnaissance automatisé de formes connues a été développé et breveté afin de recaler le modèle $3 \mathrm{D}$ connu de chaque ACCROPODETM $\mathrm{II}$ à partir de leurs faces visibles dans l'enveloppe de points. 


\section{XVI èmes Journées Nationales Génie Côtier - Génie Civil \\ Le Havre 2020}

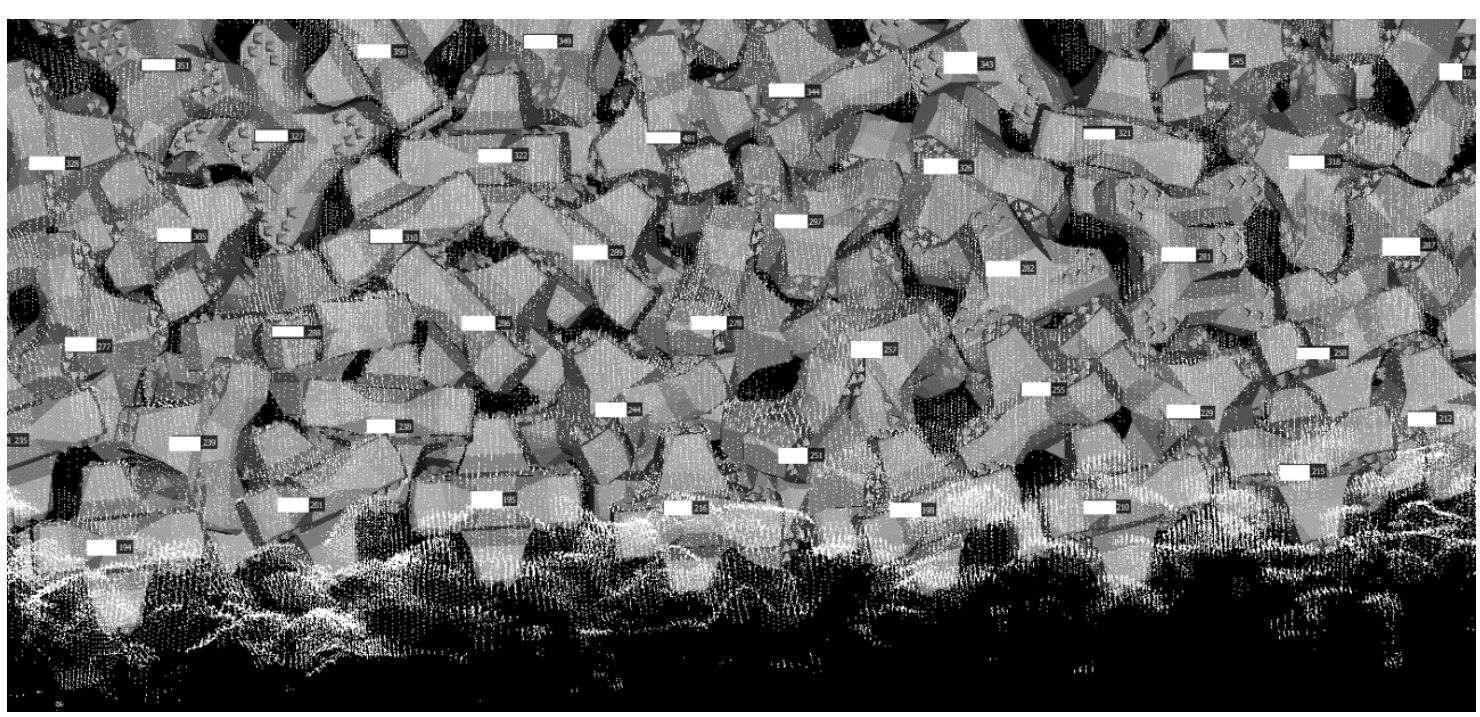

Figure 5. Etape 2 - Création du jumeau numérique 3D et numérotation automatique.

Les pourcentages de reconstruction automatisés obtenus sont de 85 à $99 \%$ en fonction de la qualité des données acquises (résolution de points, qualité de filtrage et de précision en position). Les blocs restants sont ensuite calés de manière semi-automatique, c'est-à-dire un positionnement manuel du bloc approximatif dans l'enveloppe de points et une convergence vers sa position finale par l'algorithme. La distance entre l'enveloppe et les blocs recalés est inférieure à deux centimètres sur la majorité des points du nuage comme montré ci-dessous. Ces valeurs sont cohérentes avec la précision relative du système d'acquisition.

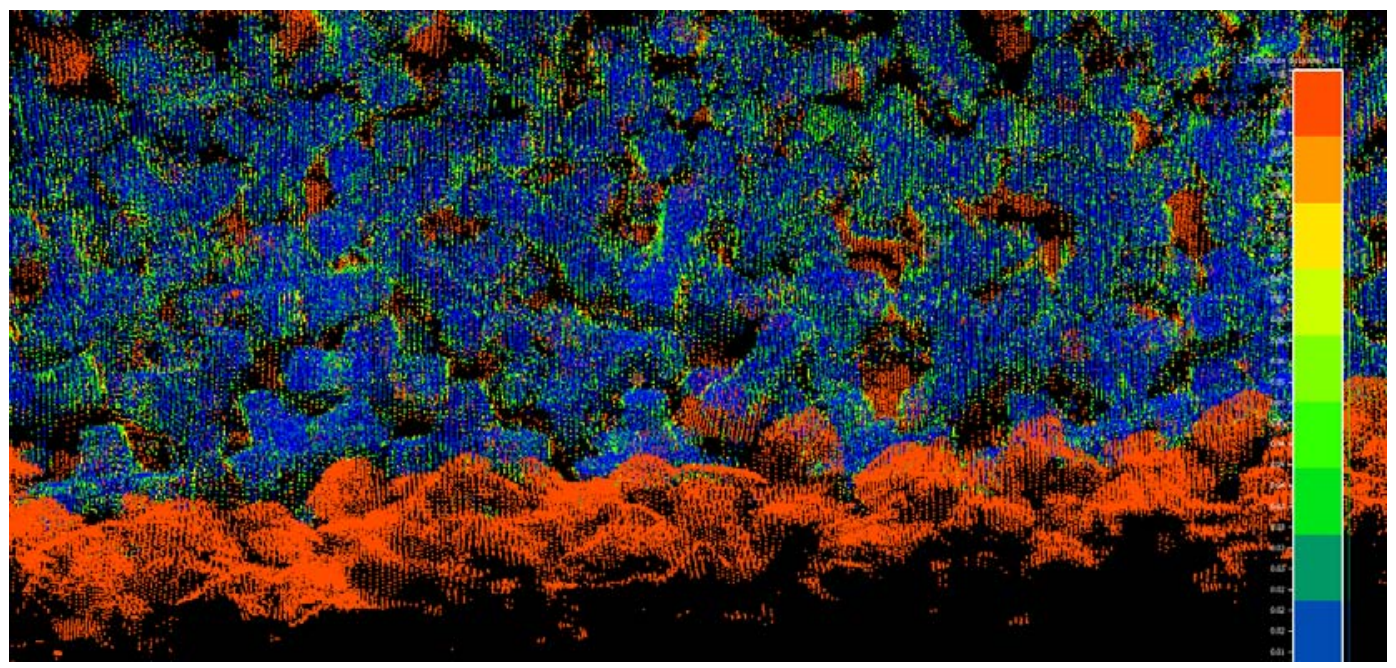

Figure 6. Etape 3 - Contrôle qualité du modèle 3D-distance entre le jumeau numérique et le nuage de points initial (bleu : inférieur à $2 \mathrm{~cm}$ ). 


\section{Thème 3 - Instrumentation, mesures, imagerie et télédétection}

Pour chaque bloc recalé, la distribution de la distance entre les points au voisinage et le modèle 3D du bloc est calculée. Des critères statistiques ajustables par l'utilisateur sont utilisés pour filtrer automatiquement les blocs valides.

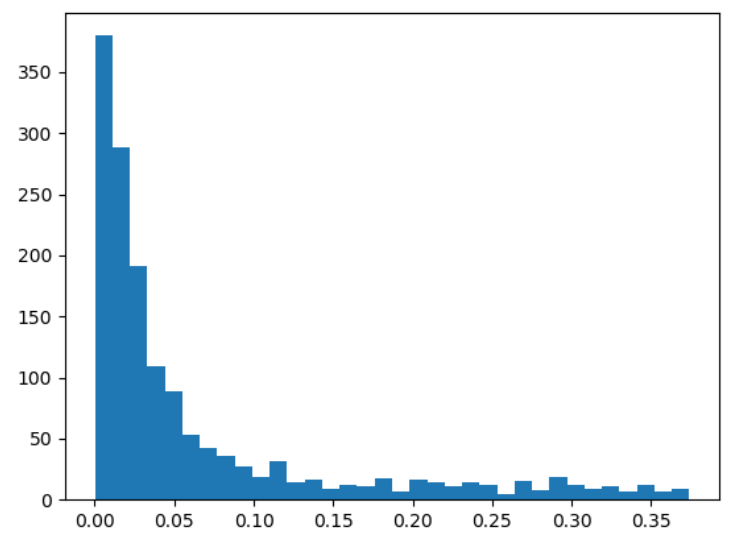

Figure 7. Histogramme de répartition de distance - abscisse : distance du point au bloc recalé /ordonnée : nombre de points par tranche de distance (tous les $1 \mathrm{~cm}$ ).

Des tests de robustesse du recalage ont été réalisés en faisant des différentiels temporels entre modèles 3D dans le temps. Il a été observé que d'un modèle 3D à l'autre, l'évolution de la digue reste cohérente. Il n'a pas exemple jamais été observé de bloc qui remonte la pente de sous-couche sur une date ultérieure ou de bloc en butée de pied qui se serait décalé latéralement de plusieurs centimètres.

L'algorithme de recalage est robuste aux éventuelles perturbations de la forme de l'enveloppe. Par exemple dans le cas d'un bloc dont une oreille est cassée, l'algorithme fonctionne tout de même s'il y a suffisamment d'autres points dans l'enveloppe externe visible du bloc pour réaliser le recalage. L'existence d'une oreille cassée sur la face externe sera alors identifiée lors de la génération du différentiel entre le modèle $3 \mathrm{D}$ et le nuage de points. La NRL étant un ouvrage récent, il n'a pas été réalisé dans le cadre du projet d'essai de recalage sur une digue présentant une forte colonisation marine.

L'algorithme génère un fichier OBJ géoréférencé et orienté pour chaque bloc recalé et le jumeau numérique est ensuite analysé sur le logiciel Cloud Compare.

Le principal avantage de l'outil est sa capacité à analyser n'importe quelle digue sur laquelle des données bathymétriques et/ou aériennes sont disponibles même si aucune donnée de position ou d'orientation des blocs n'a été enregistrée par des outils d'assistance à la pose lors de la construction, car il ne nécessite pas d'information de position initiale du bloc dans son enveloppe.

Le temps de traitement automatique sur une station de calcul type Thinkstation P620 est de $7 \mathrm{~h}$ pour le recalage automatique de $100 \mathrm{ml}$ de digue depuis un nuage de points fusionné bathymétrie et drone aérien. En fonction du taux de recalage automatique obtenu sur un tronçon, le temps de recalage semi-automatique varie entre $2 \mathrm{~h}$ et $7 \mathrm{~h}$ sur le même tronçon. 


\section{XVİ̀mes Journées Nationales Génie Côtier - Génie Civil \\ Le Havre 2020}

Le temps d'application des filtres de contrôle et de présentation des données pour remise au client est de $4 \mathrm{~h}$. Le temps moyen de génération du modèle 3D complet pour un tronçon de $100 \mathrm{ml}$ est donc inférieur à 3 jours ouvrés, moins dans le cas d'un ouvrage continu.

\subsection{Contrôle des règles de pose}

Le contrôle de qualité de pose sont normalement réalises par les ingénieurs en charge du contrôle interne de manière manuelle bloc par bloc sur la base de l'analyse des vidéos plongeur et des nuages de point. Des filtres automatiques ont alors été implémentés afin de caractériser la qualité de l'assemblement des blocs selon les critères définis par le concepteur et d'assigner un code couleur à chaque bloc en fonction d'un critère de pose défini tel que la qualité d'imbrication des blocs entre eux par exemple.
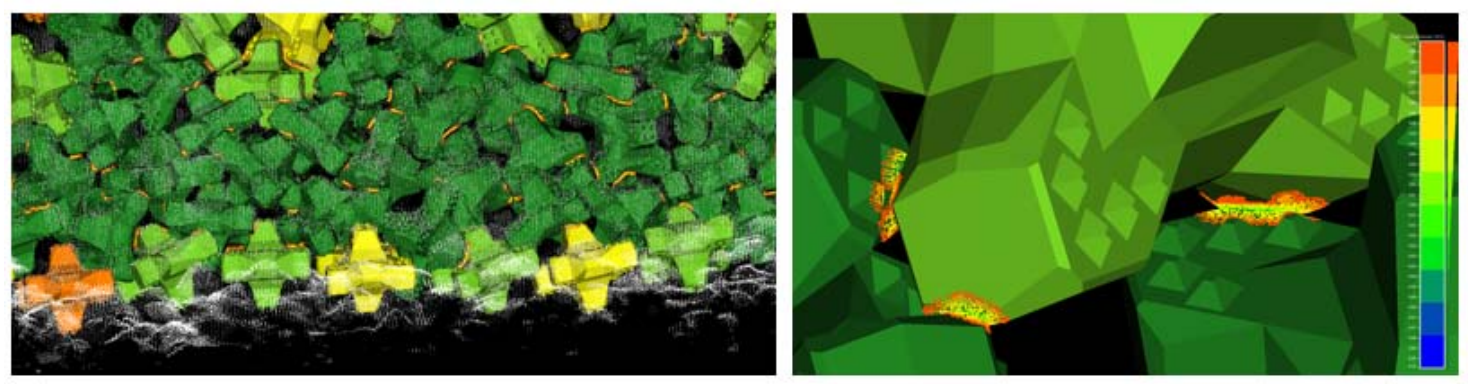

Figure 8. Etape 4 - Caractérisation de la qualité de l'assemblage (critère : nombre de contact de chaque élément avec ses voisins).

Des développements sont en cours avec le concepteur des blocs afin d'ajouter et de valider des filtres de contrôle automatique notamment sur la densité et l'orientation de pose évoqués dans le chapitre 2.1. L'objectif à terme du projet est d'aboutir à un outil de contrôle complet d'une digue qui valide sur la base du modèle $3 \mathrm{D}$ généré toutes les règles de pose de manière automatique et identifie la localisation des défauts de pose de l'ouvrage sans intervention manuelle humaine afin de sécuriser la réception de l'ouvrage pour le concepteur ou l'installateur.

\subsection{Suivi temporel de l'ouvrage}

Afin de réaliser des suivis temporels d'ouvrage durant leur durée de vie, la méthode classique consiste à superposer les nuages de points d'une date à l'autre. Cette méthode peut s'avérer difficile à analyser car la superposition de nuages de points issus d'une digue devient vite illisible. En comparant les modèles 3D obtenus par des acquisitions de nuages de points réalisés à des temps successifs de la vie de l'ouvrage, tel qu'entre la réception de l'ouvrage et après un évènement extrême par exemple, SEABIM permet de réaliser des calculs de déplacement de chaque élément. Ces informations sont ensuite représentées graphiquement, afin qu'elles soient facilement interprétables par tous les interlocuteurs 


\section{Thème 3 - Instrumentation, mesures, imagerie et télédétection}

du projet, par l'assignation d'un code couleur en fonction du déplacement par rapport au modèle de référence.

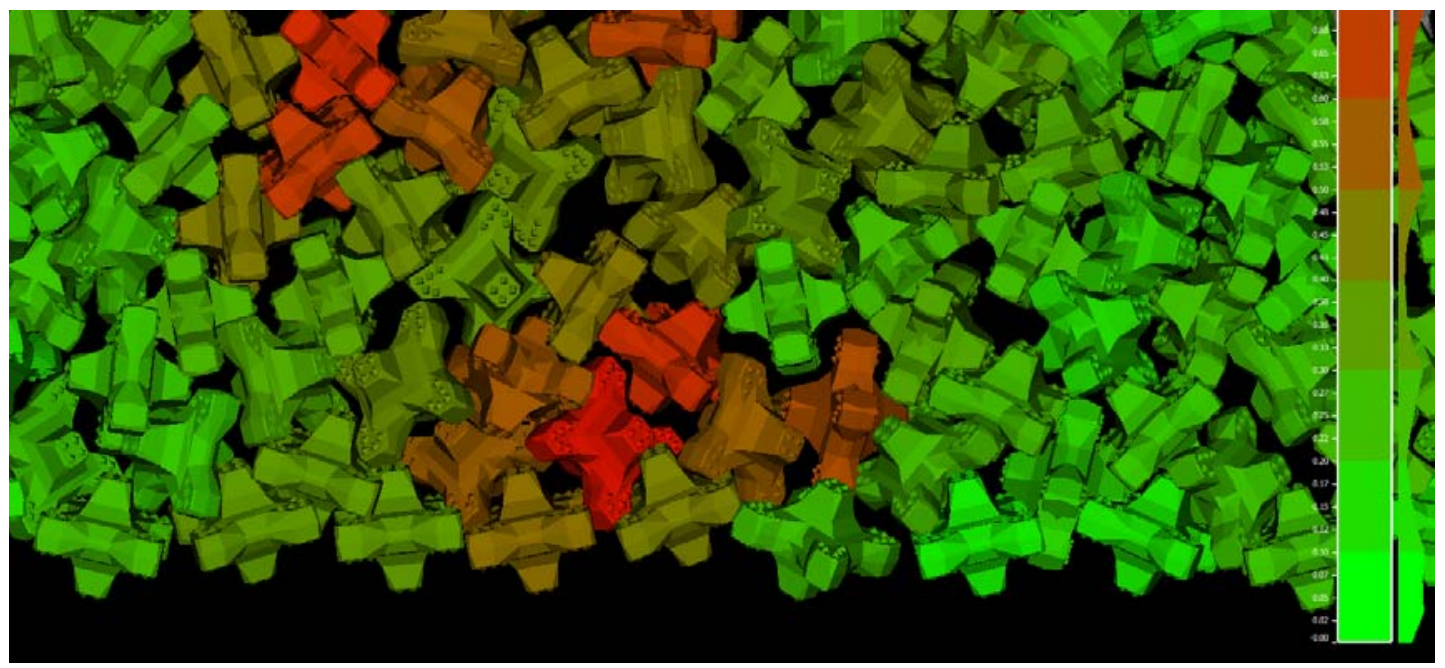

Figure 9. Etape 5 - Analyse temporelle de déplacement de la structure de digue(rouge : déplacement du centre de gravité supérieur à $1 \mathrm{~m}$ - données simulées pour essais, hors projet NRL).

\section{Conclusion}

SEABIM est un outil innovant et versatile de numérisation du suivi de digue monocouche en blocs béton préfabriqués qui permet une analyse objective et simplifiée de l'état d'un ouvrage dans le temps par tous les acteurs impliqués (maitre d'ouvrage, maitre d'œuvre, entreprise, assurances, experts judiciaires). L'outil qui a été appliqué par le groupement Digues sur le projet NRL à La Réunion sur une part significative de l'ouvrage dans une première phase de déploiement opérationnel en 2019 a permis de réduire drastiquement le temps d'analyse des données de digues par les ingénieurs en charge du contrôle interne et de réduire les coûts d'inspection d'ouvrage en ciblant plus précisément les zones à contrôler en détail. L'outil SEABIM s'intègre logiquement dans les démarches BIM et DOE (Dossier des Ouvrages Exécutés) numérique qui s'imposent progressivement aux projets maritimes.

\section{Références}

CLI (2018). Extrait des spécifications techniques ACCROPODE ${ }^{T M}$ II -- Note de présentation.

https://www.concretelayer.com/sites/default/files/2019-05/Spe\%CC\%81cifications\%20ACCRO_II_COURTES_Version\%20E_FR.PDF 